\title{
Perspective
}

PERSPECTIVE Actualité en histoire de l'art

2| 2020

Danser

\section{Kinesthésie plurielle : danse, esthétique et agentivité}

Une discussion entre Emmanuel Alloa et Carrie Lambert-Beatty, menée par Sarah Burkhalter

\section{Emmanuel Alloa, Sarah Burkhalter et Carrie Lambert-Beatty}

Traducteur : Michèle Veubret

\section{(2) OpenEdition}

\section{Journals}

Édition électronique

URL : https://journals.openedition.org/perspective/20271

DOI : 10.4000/perspective.20271

ISSN : 2269-7721

Éditeur

Institut national d'histoire de l'art

Édition imprimée

Date de publication : 30 décembre 2020

Pagination : 73-84

ISBN : 978-2-917902-90-5

ISSN : $1777-7852$

\section{Référence électronique}

Emmanuel Alloa, Sarah Burkhalter et Carrie Lambert-Beatty, « Kinesthésie plurielle : danse, esthétique et agentivité », Perspective [En ligne], 2 | 2020, mis en ligne le 30 juin 2021, consulté le 29 juillet 2022 URL: http://journals.openedition.org/perspective/20271; DOI : https://doi.org/10.4000/perspective. 20271 


\title{
Kinesthésie plurielle : danse, esthétique et agentivité
}

\author{
Une discussion entre Emmanuel Alloa \\ et Carrie Lambert-Beatty, \\ menée par Sarah Burkhalter
}

Sonder la spécificité de la danse en regard de l'art pictural invite à se pencher sur la kinesthésie, la faculté proprioceptive engagée dans l'exercice du mouvement dansant et dans l'appréciation du spectacle dansé. La pratique comme la perception du danser mobilisent un sensorium tactilo-moteur que la psycho-physiologie européenne identifie, de façon chirurgicale et anatomique, au cours de la seconde moitié du XIX ${ }^{\mathrm{e}}$ siècle. Attentive à la psychologie expérimentale qui en résulte au tournant du $\mathrm{XX}^{\mathrm{e}}$ siècle, l'avant-garde chorégraphique - considérée dans un temps long et nuancé reliant les pratiques de Lö̈e Fuller à celles de Rudolf von Laban, y compris les performances de Monte Verità, du futurisme, de Dada et du Bauhaus - incorpore les conclusions de celle-ci pour renouveler son esthétique, sa gestuaire, son expressivité ainsi que son rôle de tonique artistique, évolutionniste ou sociétal. Le sens musculaire du mouvement, alors caractérisé en contrepoint au sens strictement oculaire, promet d'accéder à un réservoir de figures et de dynamiques corporelles dont l'accent porte sur le processus, non sur la forme définitive - un paradigme qui ressurgit dès les années 1950 dans les ateliers d'Anna Halprin en particulier, après s'être progressivement codifié au fil d'une première génération de pratiques de la danse moderne allemande et américaine (fig. 1). Aujourd'hui, la neuro-esthétique informe régulièrement la recherche chorégraphique, attestant de la formation d'une préoccupation constante de la danse au sujet du sens de la motilité en tant qu'agent formel, mais aussi en tant que continuum sensoriel et perceptif entre interprètes et spectateurs.

Si l'on tient à identifier les résonances ou les indifférences entre les médiums performatif et plastique, il convient de s'interroger sur les modes de perception que chacun d'entre eux installe. Ainsi sont ici posées la question de l'émotion esthétique, qui appelle celle de l'empathie kinesthésiquel, à nos intervenants actifs, pour l'une, dans le champ de l'histoire de l'art, pour l'autre, dans celui de de la philosophie. Il en émane notamment un appel à résister aux définitions essentialistes pour questionner les conditions historiques, sociales, culturelles et institutionnelles spécifiques qui induisent, ou au contraire inhibent, une logique empathique entre l'œuvre (ou l'événement) et son public. S'il paraît évident, par exemple, que l'empathie kinesthésique ne repose 


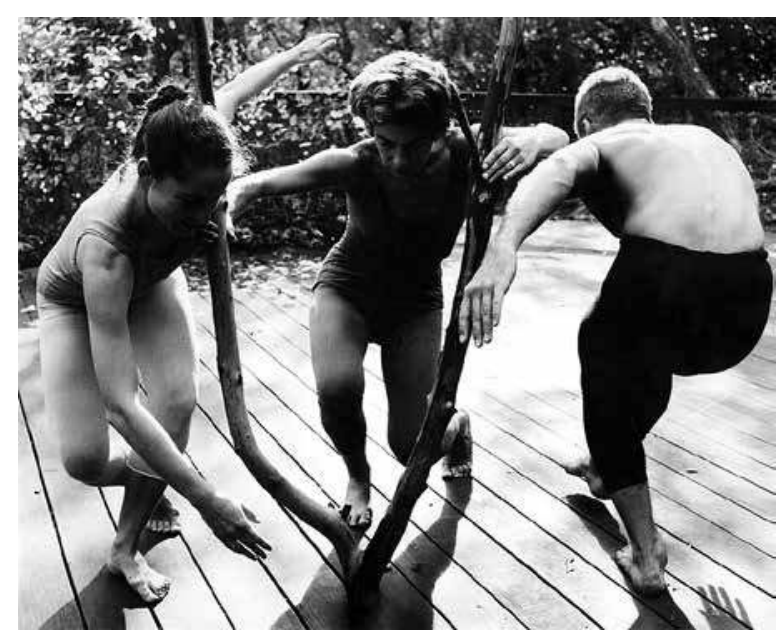

1. Warner Jepson, Anna Halprin, A. A. Leath, et Simone Forti dans The Branch d'Anna Halprin, vers 1957, San Francisco, Museum of Performance + Design, Anna Halprin Digital Archive. ni exclusivement, ni systématiquement sur le regard et le visible, mais appelle bien des sensibilités aurales, olfactives, rythmiques et spatiales, quelle agentivité assume alors le public devant de telles productions qui esquivent la représentation visuelle du mouvement tout en lui dressant une scène ?

Les échanges retranscrits ici et traduits de l'anglais ${ }^{2}$ entendent offrir des repères épistémologiques à une histoire de l'art intégrative des pratiques dansées et chorégraphiques. Partant du principe que la kinesthésie se présente comme un vecteur de prédilection de cette interdisciplinarité, dont une convergence première se joue dans les débats esthétiques, la conversation qui suit en déplie les manifestations plurielles et s'interroge, in fine, sur les paramètres et la portée de la présence en art.

[Sarah Burkhalter]

\begin{abstract}
- Sarah Burkhalter. L'émotion esthétique est fondamentalement kinesthésique, puisque tout ce que l'œil rencontre appelle des mouvements internes qui balayent l'arc de l'attraction ou de la répulsion, obligeant le spectateur ou la spectatrice à s'intéresser à l'objet, au site ou à la danse en fonction de son expérience, de son histoire et de ses aspirations. Ces réponses peuvent rester au niveau des zones stimulées dans le cerveau, sans générer de véritables gestes, mais elles puisent dans un réservoir de dynamiques, de figures ou de mouvements incarnés. Si l'empathie kinesthésique informe en effet l'opération basique d'appréciation de l'art visuel, qu'il soit de l'ordre de la performance ou de l'objet, comment se traduit-elle dans notre manière de pratiquer l'histoire de l'art et la philosophie de l'art ?
\end{abstract}

- Emmanuel Alloa. On peut sans doute aborder la question sous différentes facettes. En soulignant le caractère intrinsèquement kinesthésique de l'émotion esthétique, nous étayons une intuition déjà offerte par le langage même, c'est-à-dire que toute émotion tient bien de la motion : être ému, c'est être mû. Dans la plupart des langues, la sémantique de l'émotion comporte cette dimension de transport, parfois avec des effets spectaculaires comme dans les cas de forte colère ou de profonde tristesse. Les émotions esthétiques sont souvent considérées d'un niveau d'excitation moindre par rapport à ces états existentiels, mais elles supposent elles aussi une sorte de transport : les expériences esthétiques peuvent être émouvantes et saisissantes et, au sujet des œuvres d'art, on prétend qu'elles " élèvent » l'esprit ou, dans le cas contraire, qu'elles " ébranlent » les certitudes et "brisent » les structures perceptuelles. Ces ingrédients dynamiques réaffirment l'intime connexion entre l'aisthêsis et la kinêsis, et ce en un sens qui n'est pas que métaphorique. D'importantes découvertes neurologiques, telles que les "neurones miroirs", viennent aujourd'hui appuyer le courant de la "neuro-esthétique ", associé à des chercheurs comme David Freedberg ou Vittorio Gallese, apportant ainsi de l'eau au moulin de la phénoménologie et de l'esthétique au sens large, quand celles-ci insistaient sur le fait que la perception est indissociable du mouvement ${ }^{3}$. 
La sensorialité impose une chorégraphie permanente à notre schéma corporel, qui oriente nos mouvements d'approche vers les objets ou au contraire de prise de recul, mouvements permettant d'en faire le tour ou de les aborder de biais. Dans cette optique, en tant que pratique kinesthésique, la danse repose sur cette condition fondamentale de toute esthétique mais l'élève également à un niveau réflexif avancé. Au début du XX⿳⺈冂大 siècle, le psychiatre et phénoménologue Erwin Straus fut l'un des rares théoriciens à conférer un rôle crucial à la danse dans ses recherches sur l'incorporation et la kinesthésie ${ }^{4}$ (par contraste, et aussi surprenant que cela puisse paraître, on chercherait en vain un quelconque développement approfondi sur la danse dans les textes de Merleau-Ponty). Straus distingue la danse des mouvements utilitaires, ciblés : toute danse possède une forme d'autonomie, de valeur intrinsèque, puisqu'elle déduit l'expression gestuelle d'une puissance motrice plus fondamentale, caractérisant tout être conscient et incarné. La danse génère donc simultanément une expansion de l'« espace corporel » (Leibraum) afin d'embrasser l'« espace environnant » (Umraum), tout en produisant un retour autoréférentiel sur elle-même, tel un mouvement qui n'aurait pas d'autre portée que l'épanchement d'une joie motrice, affranchie de toute autre fonctionnalité. Straus appelle cela le «symbolisme » de la danse, mais il s'agit d'un symbolisme très particulier, puisqu'il relève de la symbolisation du non-symbolique, de ce qui n'exprime rien si ce n'est la capacité d'expression elle-même ${ }^{5}$. Voilà sans doute une explication du fait que certains philosophes aient pu soupçonner une affinité intime entre la pensée et la danse, depuis Friedrich Nietzsche et Paul Valéry jusqu'à, plus près de nous, Giorgio Agamben ou Alain Badiou.

- Carrie Lambert-Beatty. Je voudrais commencer par la dernière partie de votre question : comment la compréhension d'une empathie kinesthésique se traduit-elle dans des actes de production culturelle, y compris dans la production d'une histoire de l'art ? Ceci me fait penser à une réflexion de Joseph Roach, en 2010, sur le projet qu'il animait depuis quatre ans avec Emily Coates à Yale University. Derrière le lancement de leur World Performance Project, il y avait eu une hypothèse : "la kinésie est la nouvelle mimêsis. Comme les arts prolifèrent au sein des langages médiatisés et multiculturels de l'espace transnational, le mouvement expressif devient une langue véhiculaire, la base d'une cognition affective et d'une empathie corporelle qui ont fait leur apparition. » Mais, dans le déroulement du projet, il est intéressant de constater que les résultats furent mitigés - tout comme leurs sentiments. Ils ont dû reconnaître que, au début des années 2000, I'« espace transnational » était en fait l'espace du capital mondialisé, un contexte où « une chose circonscrite et aussi durement acquise individuellement qu'un style chorégraphique peut traverser la planète avec la célérité des accessoires de luxe ou de la main-d'œuvre bon marché ». En tant qu'importateurs culturels d'un certain genre, les organisateurs ont réalisé qu'ils « tombaient dans le camp des agents de la mondialisation, ainsi que dans son malaise ${ }^{6} »$ et ils durent modifier I'hypothèse sous-jacente initiale. L'issue de leur projet était l'urgence avec laquelle il avait fini par soulever « la question des limites de l'empathie kinesthésique, à laquelle nous venons juste de commencer à répondre ${ }^{7}$ ".

Moi aussi, j'ai des sentiments partagés. L'empathie kinesthésique décrit un aspect de la manière dont les œuvres d'art forment l'expérience. Mais, en tant qu'historienne de l'art, je m'intéresse à la manière dont certaines œuvres particulières forment l'expérience, tout en agissant dans des conditions sociales, institutionnelles et culturelles différentes. II ne s'agit pas d'écarter la possibilité d'une compréhension de l'entité abstraite ou idéale qu'est I'« empathie kinesthésique », ou bien de la propension universelle à cette expérience qui nous est accordée par les effets de l'histoire évolutionniste sur la structure du cerveau humain. De mon point de vue, cependant, ces données sont intéressantes dans la mesure où elles 
nous permettent de mieux percevoir et décrire des occurrences de leur représentation. Et ces derniers concepts sont intéressants en retour dans le cadre de projets d'interprétation culturelle et historique : quand et où l'empathie kinesthésique est-elle accentuée ou relâchée, réprimée ou encouragée ? Vers ou contre quoi est-elle dirigée ? Pourquoi devient-elle problématique à certains moments et fonctionne-t-elle sans discussion à d'autres ? Pourquoi, au début du siècle dernier, les penseurs ont-ils soudainement eu besoin d'autres mots - Einfühlung, « empathie »- pour décrire ces occurrences?

- Sarah Burkhalter. En regardant une œuvre d'art, sommes-nous des danseurs en puissance? En regardant de la danse, sommes-nous des artistes visuels en devenir?

- Emmanuel Alloa. L'oculométrie scientifique nous apprend que ce que nous prenons pour un regard soutenu consiste en fait en une "danse de la pupille » : I'œil ne voit que par un mouvement alterné de saccades et de fixations. La persistance de l'objet, aussi étrange que cela puisse paraître, n'est acquise que par des acrobaties perceptuelles. Sans que nous nous rendions compte, nos yeux sont constamment en mouvement. Même pendant le sommeil, où la phase appelée paradoxale (ou REM, de Rapid Eye Movement) consiste en de rapides mouvements oculaires derrière les paupières closes. Ce fait n'est d'ailleurs pas réservé à I'humain ; Lucrèce évoque déjà les chiens qui, pendant leur sommeil, remuent les pattes au gré du flux de leurs rêves ${ }^{8}$. Il pourrait donc y avoir quelque raison à parler d'une danse des yeux, tandis qu'inversement, dans le célèbre film de Hans Namuth sur Jackson Pollock, c'est le peintre qui est mis en scène comme un danseur, se déplaçant frénétiquement autour de la toile pour exécuter ses drippings.

Entre la danse et les arts visuels, les résonances sont donc multiples, et on n'aurait pas trop de peine à prolonger indéfiniment les exemples. Mais cette approximation présente également certains dangers, car elle pourrait effacer d'autres spécificités du geste dansé. J'ai toujours été frappé qu'une personne comme Nietzsche, dont la pensée est si profondément modelée par la musique, ait eu une attirance si forte pour la danse, à l'inverse de ses rapports plutôt distants avec les arts visuels. Je dois dire qu'il y a une déclaration de Nietzsche qui m'a toujours interpellée : «Je ne peux croire qu'à un Dieu qui saurait danser ${ }^{9}$. " Pour un philosophe, explique-t-il dans le Gai Savoir, il ne pourrait y avoir d'autre idéal que de devenir danseur. "La danse est en effet son idéal, son art également, enfin aussi son unique piété, son "service divin"10. » Lorsqu'il insiste sur les rituels religieux de la danse, où la célébration se fait " par les pieds », Nietzsche baisse les yeux pour fouler le sol du regard, se détournant au passage de la tradition néoplatonicienne de l'idea céleste qui a si longtemps façonné notre conception des arts, vers quelque chose de plus rythmique et peut-être même tellurique, bref, une connexion au terrestre. Après tout, rappelons-nous que pour Nietzsche, le Gai Savoir était aussi un savoir de Gaïa, de la Terre.

- Sarah Burkhalter. Que se passe-t-il lorsque l'œuvre d'art, dans le cas de la danse, soutient votre regard?

- Carrie Lambert-Beatty. Certains diront que c'est ce que fait toujours l'œuvre d'art. (J'ajouterais : à certains moments plus qu'à d'autres.)

- Emmanuel Alloa. Oui, mais je ne suis pas certain que la métaphore visuelle, trop séduisante, soit d'une réelle utilité dans ce contexte. Certes, nous connaissons bien l'idée d'un « objet qui nous regarde ", qui nous vient d'une tradition de la pensée des images. Walter Benjamin 
a décrit les objets " auratiques", qui fixent de leur regard les observateurs, comme dotés de la capacité de produire un réel "battement des yeux ", un Augenaufschlag, un dessillement oculaire nous obligeant à lever le regard. En anglais, ça ne marche pas tout à fait, mais en français on peut dire ça me regarde, la chose m'interpelle ou bien me concerne; avec le double sens d'une observation et d'un enjeu personnel. Paul Valéry a trouvé pour ce double mouvement, qu'il a aussi parfois appelé " chiasme ", une formule devenue célèbre : « Les choses que je vois me voient autant que je les vois ${ }^{11}$. » Tout cela est bien connu et je n'ai pas besoin de le rappeler, mais je veux quand même souligner le fait que le chiasme des regards a été principalement associé aux arts visuels et surtout à la peinture. Même la réception du fameux vers de Rilke dans "Sur le torse archaïque d'Apollon » a conduit à une sorte $d^{\prime}$ ' illustration », en transférant une sculpture en trois dimensions en une image plate. Dans ce poème de 1918, vous vous en souvenez, Rilke entreprend de décrire la sculpture antique comme une sorte d'Argos Panoptès ; il est privé de tête mais chaque parcelle de son torse regarde le spectateur, « car il $n^{\prime} y$ est de point qui ne te voie ${ }^{12} »$. Or au cours du $X X^{e}$ siècle, ce trope de l'œuvre qui nous regarde - c'est un élément frappant - a été transféré de la sculpture à la peinture, pour se rattacher désormais presque exclusivement au tableau. Peut-être estce parce que, dans notre esprit, le " croisement des regards » requiert une sorte de face-à-face.

D'où une certaine réticence à considérer la danse comme une forme artistique qui solliciterait avant tout notre regard. Dans la plupart des pratiques chorégraphiques qui me viennent à l'esprit (celles de la danse moderne et postmoderne certainement, mais même celles du ballet classique), la frontalité est déjà brisée par l'utilisation latérale de la scène et de l'espace au sens large. La danse nous force à nous rapprocher de l'une des dimensions les plus énigmatiques de notre existence incarnée : celle de la profondeur. Un corps dansant ne se contente pas de remplir un espace spécifique (la localisation de la res extensa),

2. Daniel Dobbels, Est-ce que ce qui est loin s'éloigne de l'être humain? Paris, théâtre des Abbesses, 2003.
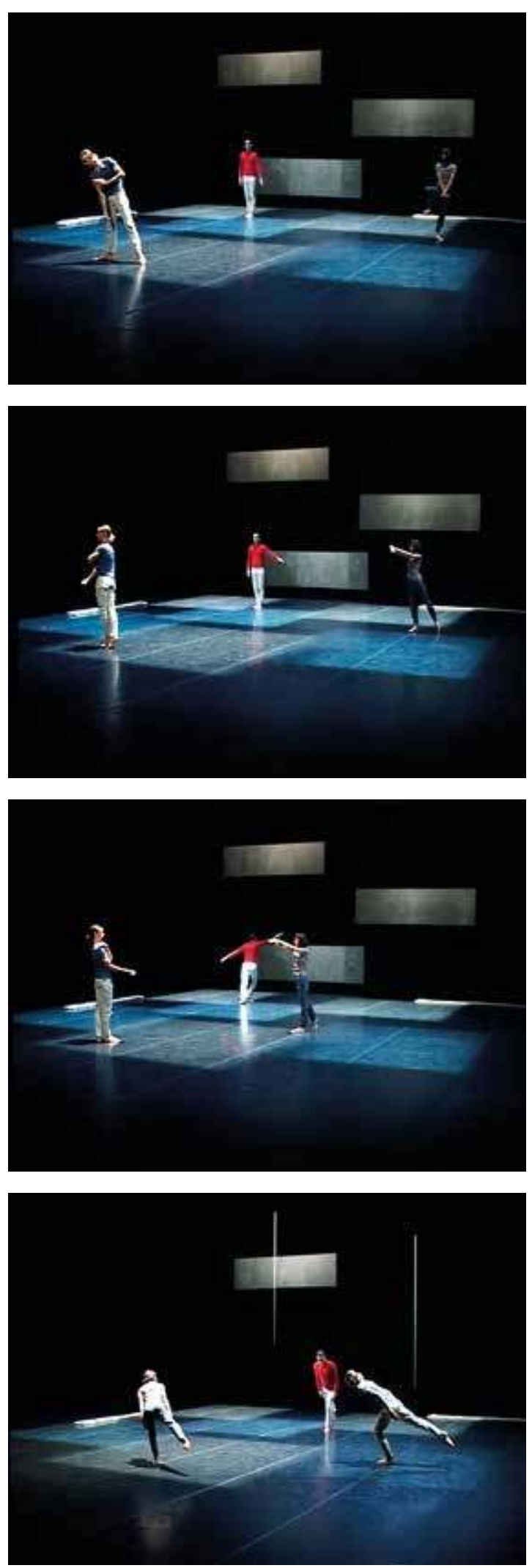


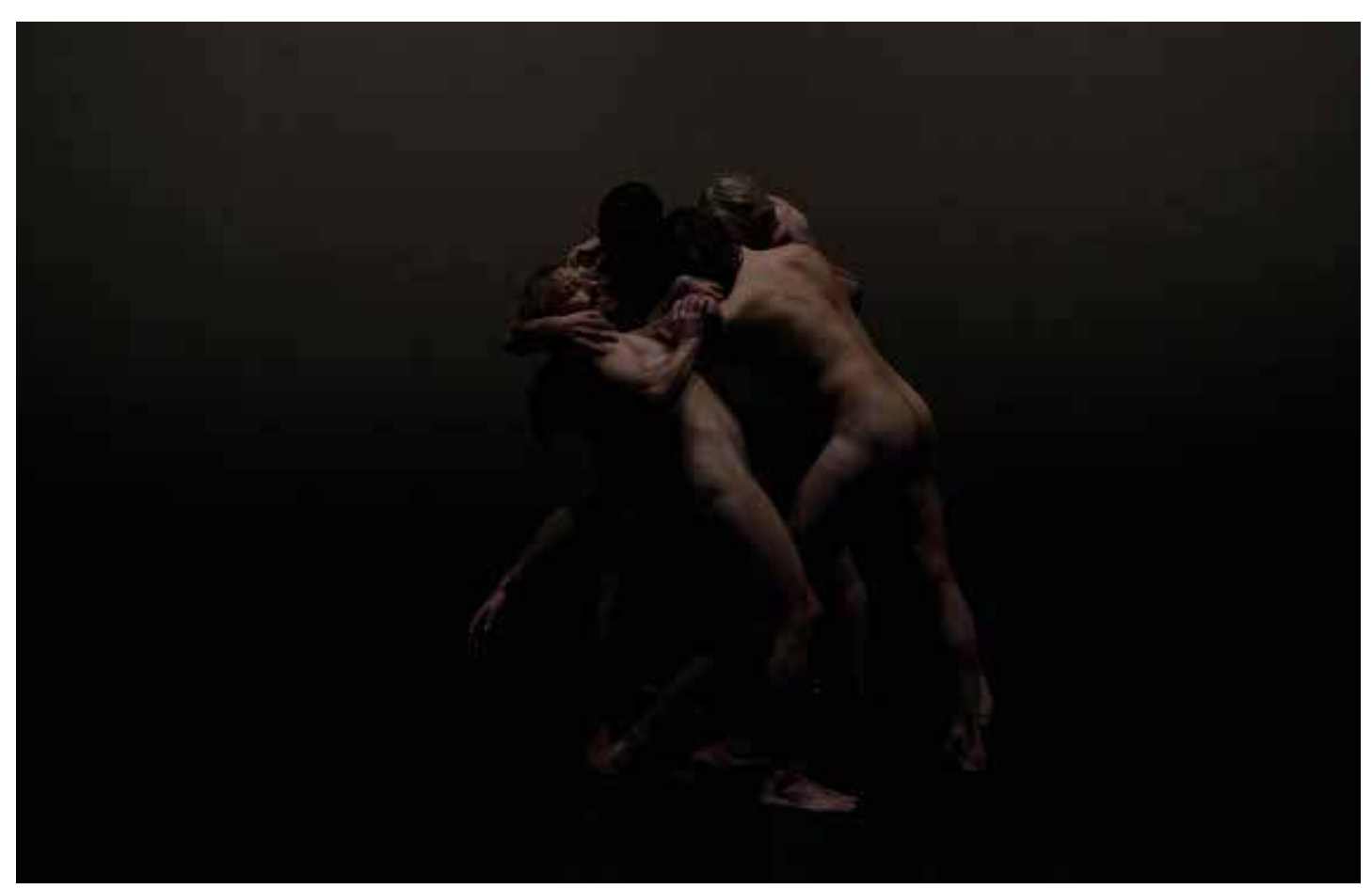

3. Pascal Rambert et Yves Godin, Memento Mori, 2013. sa gestuelle le porte vers un espace actuellement inoccupé, vers le vide comme pré-condition de tout parcours et de toute traversée. Les chorégraphes, de Steve Paxton, Yvonne Rainer et Trisha Brown jusqu'à Sasha Waltz et Daniel Dobbels (fig. 2), ont toujours insisté sur le vide spatial profondément incertain

et exploratoire qui entoure tout mouvement dansé.

Il se peut aussi que leurs pratiques chorégraphiques recherchent plutôt une prise de conscience du potentiel de ce vide qui reste largement "invisible», dans tous les sens de ce mot. Dans certaines expérimentations radicales de danse, les danseurs peuvent même se mouvoir dans le noir pendant l'intégralité du spectacle (je pense au Memento Mori de Pascal Rambert et Yves Godin en 2013, fig. 3), obligeant alors les spectateurs à s'appuyer sur leur imagination ou sur d'autres registres sensoriels, par exemple l'odorat (lors d'une orgie de fruits mûrs qui a lieu dans l'obscurité). Alors, oui, la danse fait appel à notre registre corporel, mais mettant souvent en éveil tous nos sens (et notre sens de la motilité), s ans se restreindre au face-à-face visuel.

- Carrie Lambert-Beatty. L'une des interventions les plus remarquables dans le « mini chef-d'œuvre » Trio A d'Yvonne Rainer (1965) est que les performers détournent toujours leurs yeux du public. À un moment, lorsque le danseur doit impérativement être de face, la chorégraphie précise qu'il doit fermer ses paupières. Mais je voudrais introduire ici une œuvre plus récente dans la conversation, parce que son mode spectatoriel est étroitement relié à certains enjeux de l'empathie kinesthésique, tout en les mettant au défi. Ce fut l'une des performances les plus étonnantes que j'aie jamais vue, et pourtant je n'en ai rien vu.

Compass (2009), du tandem Allora et Calzadilla, a lieu dans une grande galerie entièrement surmontée d'une plate-forme qui devient en même temps un second plafond abaissé (fig. 4). Pour cette pièce, Jennifer Allora et Guillermo Calzadilla ont collaboré avec des chorégraphes 
et danseurs locaux qui, ayant chacun composé une nouvelle pièce, se sont relayés toutes les heures pendant les heures d'ouverture de la galerie, pour exécuter dans ce " $1^{\text {er }}$ étage » une chorégraphie que personne ne pourrait jamais voir.

Allora et Calzadilla collaborent souvent avec des artistes chevronnés - chanteurs, musiciens, gymnastes - en soumettant chaque fois l'art soigneusement perfectionné de l'interprète à des conditions nouvelles et non orthodoxes. Par exemple un pianiste joue Beethoven en se tenant à l'intérieur d'un piano à queue dans Stop, Repair, Prepare: Variations on "Ode to Joy » for a Prepared Piano (2008, fig. 5). Dans Compass, la situation est plus simple : un artiste danseur exécute une pièce pour être entendu. J'ai parlé avec un musicien qui avait joué dans la pièce pour piano, et il m'a expliqué l'effort cérébral que lui avait demandé, pendant des mois, cette position de renversement par rapport au clavier. Dans Compass, les chorégraphes danseurs doivent faire quelque chose de similaire, ainsi que le « spectateur ", à qui on demande d'écouter, au lieu de regarder, le spectacle de danse. Dans la galerie, vous pouvez choisir de suivre la source du son, ou bien rester sur place et écouter les sons qui se déplacent au-dessus de votre tête. De toute façon, si vous êtes attentifs, vous commencez à remarquer des changements significatifs dans le rythme et le motif puis, après un moment, à discerner les cercles, les carrés et les zigzags du plan horizontal de la chorégraphie. Mais cette réorientation n'est pas un travail cognitif facile. (Il y avait un café dans la cour de la galerie où j'ai fait l'expérience de cette pièce et j'ai rapidement eu besoin d'un stimulant et d'une pause.)

\section{Jennifer Allora et Guillermo Calzadilla, Compass, 2010.}
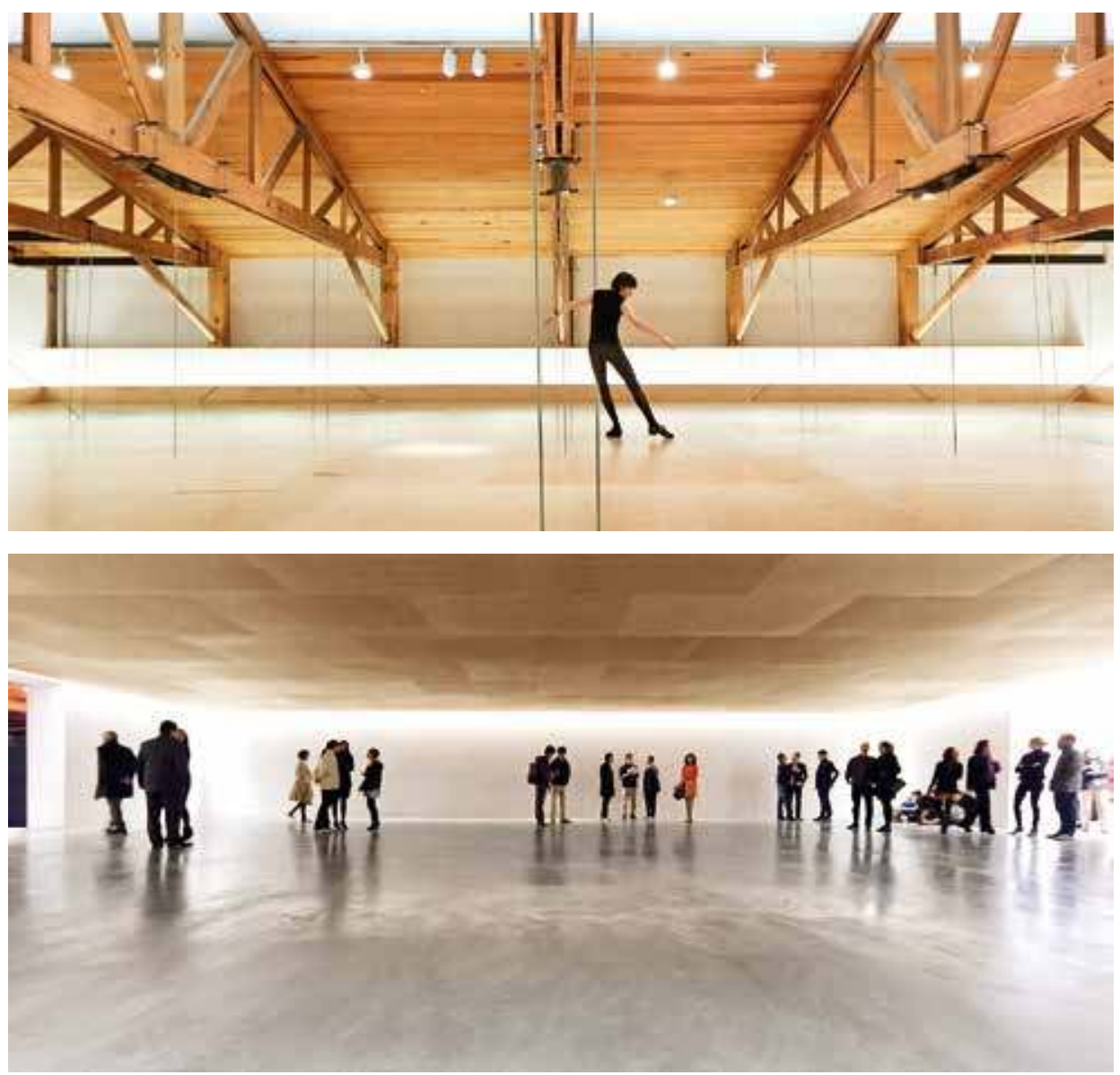


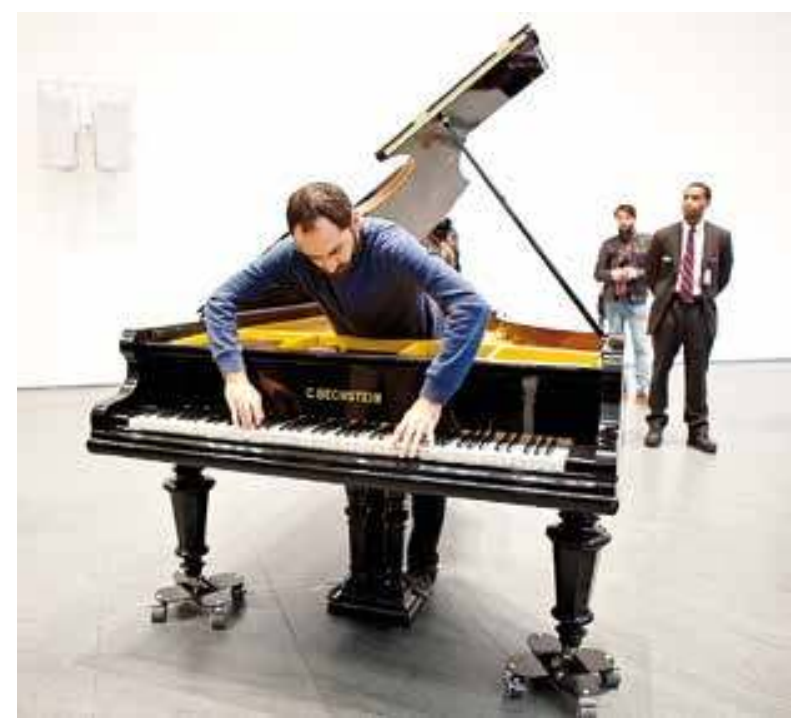

5. Jennifer Allora et Guillermo Calzadilla, Stop, Repair, Prepare: Variations on "Ode to Joy " for a Prepared Piano, No 1, 2008.
En d'autres termes, le spectateur et le danseur doivent développer de nouvelles aptitudes, et ils doivent se déshabituer de celles qui sont devenues pour eux une seconde nature. C'est sur cette attitude de spectateur, cette nouvelle seconde nature, que je voudrais attirer I'attention : pas sur les formes d'attention en elles-mêmes, qui sont codées par la marche de l'évolution, mais sur les manières dont nous les exerçons et les développons. L'identification de la neuroplasticité a produit un changement de paradigme dans la science du cerveau, et il me semble que le modèle spectatoriel de l'art proposé par Compass s'est ajusté à cette nouvelle vision, dans laquelle tout est biologique et où le biologique est conditionné et reconditionné, fondamentalement flexible. Si les exécutants de Compass devaient développer de nouveaux réseaux de neurones, et que le public tentait quelque chose de comparable (même si c'était seulement pour un bref moment) en les écoutant, alors voici la proposition : faire de l'art et le regarder sont deux activités qui requièrent une certaine habileté, et chacune d'entre elles nous transforme. Ce modèle d'art comme pédagogie sensorielle ne vient pas d'un univers différent de celui des mouvements et des sentiments « intérieurs »- peut-être même se confirment-ils l'un l'autre, à un certain degré. Mais ceci ne dépend pas de l'hypothèse selon laquelle ce que nous appelons I'« émotion esthétique " serait universelle ou immuable. Et ceci est important, en partie parce que les modèles d'empathie esthétique peuvent rapidement devenir des modèles d'intersubjectivité. Dans Compass, je ne sens pas l'effort musculaire ou cognitif du danseur ou de la danseuse, je ne m'élance pas quand il ou elle bondit, ni ne connais son plaisir ou sa douleur. Et pourtant il existe une connexion, mais sous une forme différente, entre le public et le performeur qui ont chacun reçu un travail à faire, palpable et vivifiant, ardu et transformateur.

- Sarah Burkhalter. Si l'on considère la réponse esthétique comme un phénomène déstabilisant ou instable, cela sous-entend que le public est potentiellement remué ou, au moins, mobilisé. Comment l'art de la danse et de la performance soutient-il la comparaison avec les arts basés sur l'objet en ce qui concerne l'action politique?

- Carrie Lambert-Beatty. Je ne voudrais pas amalgamer l'action politique avec les modes spectatoriels. Ce n'est pas comme si la possibilité de circuler dans une galerie était libératrice, et que le fait de rester calmement assis pendant toute la durée d'une performance était par nature déresponsabilisant; ou que l'un est fondamentalement actif et l'autre passif. Là, je crois que le modèle spectatoriel d'une seconde-nature est utile, avec la même emphase mise sur « seconde» (parce que cela implique une acculturation, des choix, un contexte) et sur «nature » (parce que cela dépend d'un dispositif corps-cerveau spécifique aux mammifères). La question porterait davantage sur la nature de l'action, ou l'agentivité, qui est postulée au nom des spectateurs. Quelle sorte de sujet perceptif une œuvre d'art invite-t-elle son public à être? Quelles sont les différences entre ce sujet et un autre qui serait aujourd'hui tout puissant? 
Et qu'exigerait-on d'un individu réel pour qu'il aille dans cette direction ? Le sujet en devenir que Compass nous invite à imaginer, ou à mettre en pratique, est un sujet neuroplastique. Ce que la science de la neuroplasticité affirme réellement est que cette seconde nature est notre nature. Nous sommes des animaux fondamentalement capables de changer.

Je trouve cette pensée réconfortante autant qu'elle donne à réfléchir. Réconfortante parce que, dans le moment politique actuel, les gens semblent de moins en moins désireux, et même incapables de changer d'avis. Elle donne à réfléchir parce qu'elle suggère la somme de travail nécessaire pour ce faire. Changer les mentalités apparaît comme une capacité qui peut être atrophiée ou développée, selon les besoins d'un moment, ou d'un producteur culturel particulier. L'empathie esthétique comprise comme première nature n'est que de peu d'utilité pour moi en ce moment. II semble en revanche important d'explorer l'idée selon laquelle on puisse apprendre toute sorte d'empathie.

- Emmanuel Alloa. C'est certainement vrai, et ce n'est pas un hasard si les avant-gardes se sont emparées de ce côté performatif de la danse pour questionner l'ensemble des normes et des pratiques sociales, depuis Isadora Duncan jusqu'à Allan Kaprow. Mais le vague désir $d^{\prime}$ « action » et de politisation que certains ressentent dans une époque d'incertitude et d'impasse politique ne devrait pas conduire à l'inverse à un néo-romantisme de la mobilisation. Souvenons-nous que la danse fut souvent utilisée comme un facteur de «mobilisation totale » prônée par les régimes autoritaristes : le régime nazi, par exemple, a commandé à Rudolf von Laban une chorégraphie à l'échelle nationale, avec plusieurs milliers de participants amateurs qui devaient se réunir au stade pour former un Volkskörper dansant, des milliers de corps synchronisés par un schéma unique. Dans ce genre de cas, la mobilisation ressemble plutôt à une mise au pas.

Par ailleurs, je m'interroge aussi sur une distinction trop facile entre les arts de la danse et les arts basés sur des objets. Pour commencer, souvenons-nous que la danse n'a jamais été un espace sans objet : sans ses "prothèses", Loïe Fuller ne serait jamais devenue le papillon admiré par Mallarmé et tant d'autres de ses contemporains. En outre, la chorégraphie n'implique pas forcément des danseurs, et même, en certains cas, elle n'implique même pas forcément des corps humains. Certains chorégraphes sont allés très loin dans cette voie : en France, Christian Rizzo est un virtuose dans l'art de faire danser les objets, tandis qu'avec Black Flags, William Forsythe est allé jusqu'à programmer un algorithme pour obtenir de deux robots qu'ils agitent deux grands drapeaux noirs (fig. 6). Il y a quelque chose de profondément terrifiant dans ces grands mâts avec leurs pavillons de textile noir flottants qui balayent les airs par un simple pouvoir mécanique, comme une sorte d'anticipation de la prise de pouvoir par les machines. Et pourtant, l'impression qui transpire de cette lente ondulation des panneaux en soie de parachute est aussi d'une beauté désarmante. On pourrait bien sûr débattre pour savoir si, avec Black Flags, Forsythe a élargi le domaine de la danse ou si son geste représente plutôt une élégie de la disparition d'un monde centré sur I'humain, désespérément obsolète par rapport à une logique de production algorithmique.

- Sarah Burkhalter. L'espace dans lequel la danse évolue et perdure peut être partagé dans le même instant ou enregistré pour un futur spectacle ou une future visualisation. Comment cela affecte-t-il les notions d'authenticité et de matérialité, à la fois de l'œuvre représentée et de l'expérience de celle-ci?

- Emmanuel Alloa. Vaste sujet. Erwin Straus, pour revenir vers lui un instant, a voulu distinguer au sein des genres artistiques des arts liés à un espace " historique » et des arts liés à un espace " présentiel », associant la danse à la présence. Évidemment, en un certain 
sens, cette distinction est pertinente, et l'exigence de présence a donné son aura à la danse comme à de nombreux autres " arts vivants ", voire même son « ontologie », pour reprendre le mot de Peggy Phelan ${ }^{13}$. Pourtant, beaucoup d'éléments viennent contredire ce désir d'une présence immédiate. Comme dans tout autre art performatif, la performance dansée n'apparaît jamais pleinement dans l'« ici et maintenant », elle ne relève jamais tout à fait d'une " première fois »; tout performeur sait bien que la performance suppose un entraînement patient, et des répétitions infinies. Même l'Impro Dance n'est jamais totalement improvisée, cela va sans dire. Richard Schechner enfonce le clou en affirmant que « la performance est "un comportement répété" [twice behaved] ${ }^{14} »$. Tout ceci questionne profondément toute notion d'authenticité, qu'elle soit naïve ou complexe. Dans le cas de la danse, il me semble qu'il y a également un problème spécifique : celui de la transmission. Contrairement aux arts classiques dits " allographiques", où un certain genre de notation permet de coder la production artistique (pensons à la musique classique européenne), en danse, les partitions chorégraphiques sont plutôt l'exception que la règle. Si l'on pense à la situation de la danse contemporaine, notamment à partir des années 1960, transmettre à un danseur l'interprétation d'une certaine pièce, c'était lui demander de s'entraîner face à des enregistrements vidéo. Et bien sûr, vous l'évoquiez, ceci entraîne également un déplacement dans la réception, puisque la plupart des grandes œuvres légendaires du performance art n'ont été vues que par un nombre extrêmement restreint de personnes, tandis que le reste n'a accès qu'à des bandes vidéo qui ont, elles, conféré une dimension auratique, inconnue jusque-là, aux performances initiales. On en trouve un exemple des plus symptomatiques dans le film sur la performance de Marina Abramović, The Artist is Present, au Museum of Modern Art de New York (2010). Tout au long de la performance, l'artiste fixe immobile les spectateurs prenant place sur une chaise face à elle. Or comment ne pas voir que cette célébration de la présence, dans tous les sens du terme, est celle d'une présence pérennisée à l'infini, puisque qu'au-delà du cercle des élus présents pendant les semaines que dura la performance, la plupart ne la connaissent que grâce au long-métrage documentaire projeté dans les cinémas. Avec ce film et son dispositif technique, Abramović, la pionnière et légende vivante dont les performances avaient pour but de contrer la logique du monument, s'érige à son tour un momument vidéographié, célébrant pour ainsi dire une présence sans fin et quasiment divine. Dans le même temps, il faut savoir qu'elle enseigne aussi à de jeunes performeurs comment rejouer ses propres pièces anciennes. Ces questions de documentation, de transmission et de reprises de performances soulèvent des questions fondamentales quant à la stabilité de cette ontologie prétendument figée de la performance, et de la danse, par extension.

- Carrie Lambert-Beatty. Les deux termes que vous proposez, authenticité et matérialité, sont intéressants à considérer, tout comme l'est la manière dont ils semblent aller de pair si facilement. On ne pourra pas dire que la matérialité d'une performance médiatisée est la même que celle d'un spectacle vivant, mais cela ne la rend pas inauthentique. Même dans les arts visuels on peut avoir une copie non authentique qui apporte abondance de matière. Peut-être est-ce à l'intersection de la matérialité et de l'authenticité que l'on trouve la présence, le desideratum constant de tant de philosophie (et aussi l'économie de l'expérience). Je pense qu'il est assez facile maintenant de se débarrasser de la discussion qui opposait la présence à la médiation dans laquelle la théorie de la performance a été autrefois tellement impliquée. (Je suis présente avec un danseur de chair d'une manière différente de ma présence à une vidéo de sa danse mais les deux sont des expériences esthétiques.) Mais ceci est plus qu'un débat universitaire suivant son cours. Le contexte médiatique a connu une transformation majeure depuis la fin des années 1990. David Joselit théorise que, dans notre " épistémologie de la recherche » contemporaine, les images s'apparentent à la monnaie ; leur pouvoir s'ajoute 


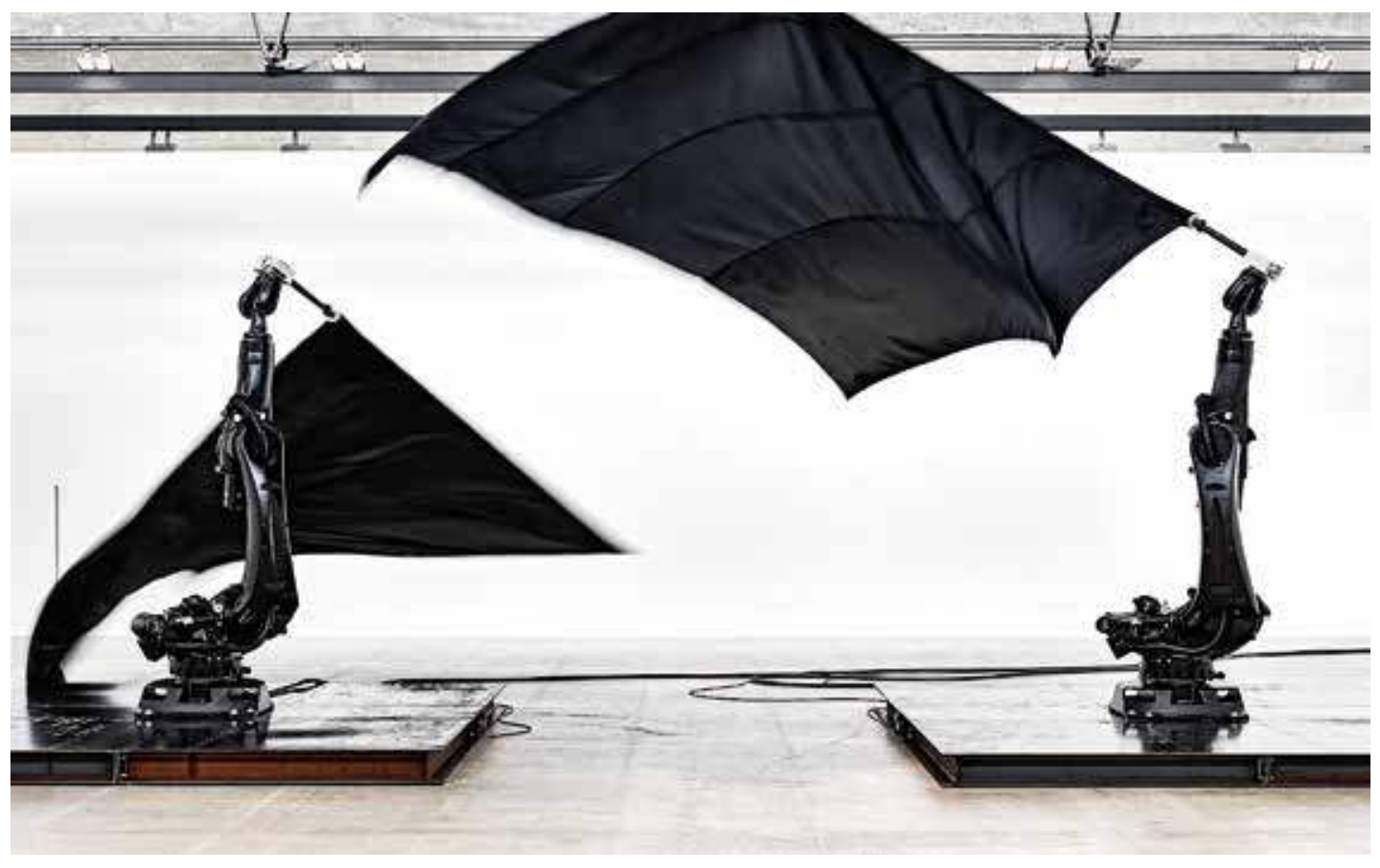

à l'algorithme avec l'intervention humaine : la boucle rétroactive des visionnages, des tweets, des commentaires et des « likes »". Lorsqu'Abramović doublait la mise sur l'aura de la performance dans The Artist is Present, c'était de notre côté de cette transformation médiatique. Ce qui s'est ensuivi au Museum of Modern Art fut soit une expérience transcendantale, soit un spectacle de supplice, selon les points de vue. Toutefois, je ne remets pas en question l'authenticité de l'expérience vécue par les fidèles, car elle n'est pas incompatible avec le fait que l'imbrication de la performance et de sa médiation était déployée dans toute sa splendeur. En effet, tout cela était un tournage élaboré mais, loin d'interférer avec la présence de la performeuse, il tenait lieu d'amplificateur d'aura ${ }^{16}$.

D'une certaine manière, ceci est lié à la précédente question au sujet de l'action politique. La présence, dans la tradition philosophique occidentale, est une aspiration métaphysique. Mais on peut également partir de l'hypothèse qu'une chose comme la présence est tout simplement... normale. Interdépendance et processus - empathie et mouvement - pourraient être l'horizon de référence et non l'exception, comme ils le sont dans de nombreuses traditions non occidentales ainsi que dans les lignées philosophiques alternatives qui fermentent au sein des dominantes. Et cette réorientation fait une grande différence. Je pèse mes mots en disant que le modèle du jardin d'Eden est loin de convenir à tout le monde, et qu'il existe des alternatives. Il s'agit de réapprendre le bon sens : exactement ce que l'art de tout genre a la capacité - et peut-être la responsabilité - de faire.

Les contributions d'Emmanuel Alloa

et de Carrie Lambert-Beatty, ainsi que les questions formulées par Sarah Burkhalter ont été traduites de I'anglais par Michèle Veubret. 


\section{Emmanuel Alloa}

Emmanuel Alloa est professeur ordinaire en esthétique et philosophie de l'art à l'Université de Fribourg. Ses recherches portent notamment sur la pensée contemporaine, la phénoménologie (Merleau-Ponty en particulier) et l'esthétique. Lauréat du prix Latsis 2016 et du prix scientifique Aby Warburg 2019, il a été commissaire adjoint de l'exposition Le supermarché des images (Paris, Jeu de Paume, 2020). Parmi ses derniers ouvrages parus : Partages de la publication (Paris, Fayard, 2020) et Dynamis of the Image. Moving Images in a Moving World (codir., Berlin, De Gruyter, 2020).

\section{Sarah Burkhalter}

Sarah Burkhalter est historienne de l'art et responsable de I'Antenne romande de I'Institut suisse pour l'étude de l'art (SIK-ISEA) à Lausanne. Docteure ès lettres, elle s'est formée à New York University et à I'Université de Genève, où elle a été chercheuse-boursière du Fonds national suisse de la recherche scientifique (FNS) et a enseigné l'histoire de l'art et de la danse aux XIX $\mathrm{X}^{\mathrm{e}}$ $\mathrm{XXI}^{\mathrm{e}}$ siècles, ses domaines de spécialisation. Elle a codirigé, avec Laurence Schmidlin, Spacescapes. Danse et dessin depuis 1962 (Zurich, JRP | Ringier, 2017) et The Postcard Dialogues. Mélanges en I'honneur de Dario Gamboni (Lausanne, art\&fiction, 2020), et prépare la publication du livre issu de sa thèse, Vers une kinesthétique. Danse moderne, arts visuels et perception (Dijon, Les Presses du réel, 2021).

\section{Carrie Lambert-Beatty}

Carrie Lambert-Beatty est historienne de l'art et professeure à Harvard University. Elle est I'auteure de Being Watched: Yvonne Rainer and the 1960's (Cambridge, Mass., MIT Press, 2008). Elle explore actuellement la "fiction épistémique " et l'art de la parafiction (quand la fiction est présentée comme un fait) depuis 1989.

\section{NOTES}

1. Parallèlement à une vaste littérature issue des champs de la physiologie et des neurosciences, nous retenons ici les écrits qui abordent l'empathie kinesthésique dans le champ des performance studies et de l'histoire de l'art. Voir notamment Susan Leigh Foster, "Kinesthetic Empathies and the Politics of Compassion ", dans Janelle G. Reinelt et Joseph R. Roach (dir.), Critical Theory and Performance, Ann Arbor, The University of Michigan Press, (1992) 2007, p. 245- 257 et Choreographing Empathy. Kinesthesia in Performance, Londres / New York, Routledge, 2011 ; Arnauld Pierre, "La danse des yeux : empathie kinesthésique et esthétique de l'"arabesque moderne" ", dans Les Cahiers du MNAM, n 102, 2007, p. 4-19;
Dee Reynolds et Matthew Reason (dir.), Kinesthetic Empathy in Creative and Cultural Practices, Bristol / Chicago, Intellect, 2012 ; Dario Gamboni, Potential Images: Ambiguity and Indeterminacy in Modern Art, Londres, Reaktion Books, 2002.

2. Les échanges entre Emmanuel Alloa, Sarah Burkhalter et Carrie Lambert-Beatty, dont ce texte est issu, ont eu lieu par mail, entre septembre et novembre 2020.

3. Vittorio Gallese et al., "Action Recognition in the Premotor Cortex », dans Brain, a Journal of Neurology, $\mathrm{n}^{\circ}$ 119.2, 1996, p. 593-609. David Freedberg et Vittorio Gallese, "Motion, Emotion and Empathy in Aesthetic Experience ", dans Trends in Cognitive Sciences, $\mathrm{n}^{\circ} 11$, 2007, p. 193-203.

4. Erwin Straus, Du sens des sens. Contribution à l'étude des fondements de la psychologie, G. Thines et J.-P. Legrand (préface et traduction), Grenoble, J. Millon, 2000 [éd. orig. : Vom Sinn der Sinne, ein Beitrag zur Grundlegung der Psychologie, Berlin, J. Springer, 1935].

5. Pour une lecture plus détaillée d'Erwin Straus, on renverra notamment aux études de Frédéric Pouillaude, Le Désœuvrement chorégraphique, étude sur la notion d'œuvre en danse, Paris, Vrin, 2009, chap. I-III, et d'Anne Boissière, "Vers une psychologie du mouvement l'espace acoustique d'Erwin Straus, entre musique et danse ", dans Insistance, 2011/1, n 5, p. 55-68.

6. Toutes les citations sont tirées de l'article de Joseph Roach, "Kinesis. The New Mimesis ", dans Theatre,

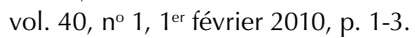

7. Roach, 2010, cité n. 6 . Toutes les citations sont issues de la p. 2 ; je souligne.

8. Lucrèce, De rerum natura, livre quatrième, 988-994.

9. Friedrich Nietzsche, Ainsi parlait Zarathoustra, I, « Du lire et de l'écrire », Geneviève Bianquis (trad. fr.), Paris, Flammarion, 1996.

10. Friedrich Nietzsche, Le Gai Savoir, § 381 «Éléments pour la question de l'intelligibilité », Patrick Wotling (trad. fr. et introduction), Paris, Flammarion, 1997.

11. Paul Valéry, OEuvres, J. Hytier (éd.), Paris, Gallimard (Pléiade), 1960, t. II, p. 729. Pour une étude détaillée de la danse chez Valéry, voir Edwige Phitoussi, Degas, Danse, Dessin de Paul Valéry, Paris, L'Harmattan, 2009.

12. La traduction de ce vers, par Jacques Legrand, est tirée de l'édition des poèmes de Rainer Maria Rilke, CEuvres 2, Poésies, Paris, Éditions du Seuil, 1972.

13. Peggy Phelan, "The Ontology of Performance. Representation without Reproduction ", dans Unmarked. The Politics of Performance, Abingdon, N.Y., Routledge, 1993, p. 146-166.

14. Richard Schechner, Between Theatre and Anthropology, Philadelphie, University of Pensylvania Press, 1985, en particulier le chap. II, " Restoration of Behavior " p. 51.

15. Voir David Joselit, After Art, Princeton, N.J., Princeton University Press, 2012.

16. J'ai traité de quelques points connexes à l'époque : "Against Performance Art ", dans Artforum, vol. 48, no 9, mai 2010, p. 208-213. 